\title{
Perinatal outcome of stage-based management of fetal growth restriction in a government tertiary care hospital in Kerala, India
}

\author{
Bhargavi Rangarajan*, Lalithambica Karunakaran
}

Department of Obstetrics and Gynecology, Government T. D. Medical College, Alleppey, Kerala, India

Received: 19 April 2021

Accepted: 17 May 2021

\author{
*Correspondence: \\ Dr. Bhargavi Rangarajan, \\ E-mail: bhargavi.rangarajan@gmail.com
}

Copyright: (c) the author(s), publisher and licensee Medip Academy. This is an open-access article distributed under the terms of the Creative Commons Attribution Non-Commercial License, which permits unrestricted non-commercial use, distribution, and reproduction in any medium, provided the original work is properly cited.

\begin{abstract}
Background: Fetal growth restriction (FGR) is the largest contributing factor to perinatal morbidity, mortality and impaired neurodevelopment. This research strives to elucidate the perinatal outcomes of stage-based management of FGR using obstetric doppler and its association with maternal sociodemographic profile.

Methods: The research was conducted among 320 antenatal women whose estimated fetal weight was $<10^{\text {th }}$ centile. Periodic follow up with Doppler was done and managed as per the stage of FGR. Perinatal outcomes were compiled.

Results: The incidence of FGR in T. D. medical college, Alappuzha was $15.23 \%$. SGA accounted for $47 \%$. The proportion of early and late onset FGR was 10.3 and $89.7 \%$ respectively. $57.18 \%$ of the newborns were admitted to NICU. The common complications were: Low birth weight-47.8\% ARDS-21\%, sepsis-9.6\%, necrotizing enterocolitis$4 \%$, hyperbilirubinemia-4.9\%. The incidence of Neonatal death and stillbirth were 1.56 and $0.3 \%$ respectively. Mothers who were underweight, inadequate weight gain during pregnancy and short inter pregnancy interval had increased risks. Hypertensive disorders of pregnancy were the commonly associated medical condition.

Conclusions: Prolongation of pregnancy even by one day results in $2 \%$ increased chances of survival of the newborn. Hence, it becomes imperative to identify the benign forms of FGR to prevent iatrogenic prematurity. Antenatal women should be screened for risk factors and undergo vigilant antepartum surveillance to bring about favourable perinatal outcome.
\end{abstract}

Keywords: FGR, Small for gestational age, Ultrasound and Doppler, Perinatal outcome

\section{INTRODUCTION}

Fetal growth restriction (FGR) represents a serious condition that can lead to increased perinatal morbidity, mortality and impaired postnatal neurodevelopment. ${ }^{1,2,4,7,9}$ There are two distinct phenotypes of FGRs-early and late onset FGR, with different onset, patterns of evolution and fetal Doppler profile. In early onset FGR, the main Doppler modifications are at the level of umbilical artery, with progressive augmentation of the pulsatility index to absent or reverse end diastolic flow. The modifications of the cerebral, cardiac and ductus venosus circulation are generally present, but with different sequences. The late onset FGR is determined by third trimester placental insufficiency that entails fetal hypoxia. ${ }^{1,2}$ The cerebro placental ratio and the pulsatility index of the Middle cerebral artery (PIMCA) seems to be the main markers for both diagnosis and obstetrical management while umbilical Doppler PI is frequently normal. Also, the sequence of Doppler alterations is neither specific nor complete. New protocols for the diagnosis and management of late onset FGR need to be implemented. ${ }^{1,2}$

Placentation is the determinant factor for developing early or late onset FGR. ${ }^{1}$ Doppler examination analyses the circulatory modifications of the fetus that can be either lesional or adaptive reactions. The two phenotypes of FGR are distinct by the moment of onset, evolution, Doppler parameters modifications and post-natal outcome. ${ }^{2}$ The 
best cut off between early and late FGR is 32 weeks in terms of perinatal outcome. , $^{1,4}$

The cerebroplacental ratio represents the ratio between the MCA pulsatility index and umbilical artery pulsatility index. In normal fetuses, it is greater than 1. It is an extremely useful tool in the assessment of vascular cerebral hemodynamics in pregnancies complicated with FGR where it realizes the quantification of brain sparing effect. $^{1,4}$

According to the classification of still birth by relevant condition at death (ReCoDe), a population-based cohort study, FGR accounts for around $40 \%$ of unknown causes of intra uterine deaths. ${ }^{1,3}$

Growth restricted fetuses have an augmented risk of morbidity and mortality. The severe morbidity is represented by intraventricular hemorrhage, bronchopulmonary dysplasia, necrotizing enterocolitis, sepsis, pulmonary hemorrhage, hypothermia and hypoglycemia. Appropriate antenatal diagnosis, treatment and timely delivery could diminish these risks significantly. ${ }^{1,3}$

FGR is among the obstetrical entities with the greatest variation in clinical practice. This results from a combination of the lack of strong supportive evidence, the complexity of the variables and indices that need to be integrated for assessing fetal deterioration, and the variable risks associated with prematurity at different gestational ages. $^{5,7}$

Although when considered as groups, there are clear differences between early and late onset forms. ${ }^{2,8}$ On an individual basis there is important overlapping of clinical features at borderline gestational ages. In addition, cases with the same gestational age at onset are often detected at different time points during gestation. Consequently, a management scheme that establishes follow up intervals and timing of delivery on the basis of fetal risks can include both early and late onset forms in an integrated fashion. ${ }^{2,8}$

The main aim behind clinical management of FGR should be firstly to distinguish FGR from SGA and secondly to ascertain whether there is risk of in utero fetal injury or death. ${ }^{2,10}$ Thus, the first step is to identify within the small fetuses the subset of SGA, because they have a normal perinatal outcome and the pregnancy can be continued till term. ${ }^{2,10}$ However the pathological forms of FGR have an increased risk of adverse outcome and stillbirth and should be managed actively once diagnosis is made. ${ }^{2,10}$

Prolongation of pregnancy towards term even by one day results in $2 \%$ increased chances of survival of the fetus. ${ }^{1}$ The current management of FGR is more subjective and individualized relying mainly on Umbilical artery Doppler. ${ }^{4}$ This results in unjustified Preterm deliveries carrying with it, its own complications. However, when
FGR is staged and managed according to the Barcelona classification, where fetal middle cerebral artery and ductus venosus Doppler parameters are taken into consideration, it allows a more objective management of FGR. ${ }^{7}$ It also results in pregnancy being continued for a longer duration resulting in improved perinatal outcomes and decreased preterm deliveries. ${ }^{4,7}$

While strong evidence is lacking to support firm recommendations on the timing of delivery, a protocol that integrates the best available evidence can help reducing clinical practice variation. One approach is to group in stages those indices or signs that are associated with similar fetal risks, since they should indicate similar follow up intervals and timing of delivery. ${ }^{1,4}$ Thus based on the existing evidence extensively discussed above and, where no evidence is available on expert opinion, it is required to profile several stages or prognostic groups, which define different management strategies. ${ }^{1,4}$

FGR is defined by an UA PI $>95^{\text {th }}$ centile or FGR using a combination of $\mathrm{CPR}<5^{\text {th }}$ centile, UtA PI $>95^{\text {th }}$ centile and an EFW $<3$ rd centile. $^{1,4}$ A remarkable proportion of ' $S G A$ ' defined by UA PI are reclassified as true FGR when the combined definition is used, particularly among late-onset FGR fetuses. ${ }^{1,4}$ Use of a lower threshold for defining pathologic FGR is supported by findings of a large, prospective observational trial (PORTO) that included over 1100 pregnancies with non-anomalous fetuses with estimated fetal weight $<10^{\text {th }}$ percentile on ultrasound examination. Only $2 \%$ of fetuses at the $3^{\text {rd }}$ to $10^{\text {th }}$ percentile $(5 / 254)$ experienced adverse perinatal outcome, while $6.2 \%$ of those $<3^{\text {rd }}$ percentile $(51 / 826)$ had an adverse outcome and all eight mortalities were in this group. ${ }^{1,12}$ The combination of estimated fetal weight $<3^{\text {rd }}$ $\%$ and abnormal umbilical artery Doppler was a strong and consistent predictor of adverse outcome: 16.7 percent of these fetuses developed intraventricular hemorrhage, periventricular leukomalacia, hypoxic ischemic encephalopathy, necrotizing enterocolitis, bronchopulmonary dysplasia, sepsis, or death. Abnormal Doppler in this study included both pulsatility index $>95^{\text {th }}$ centile and absent or reversed end-diastolic flow. An abnormal growth trajectory over time was another factor that predicted perinatal complications (e.g., preterm birth, preeclampsia, neonatal morbidity. ${ }^{1,9}$

FGR is observed in $24 \%$ of newborns, approximately 30 million infants suffer from FGR every year. The burden is mainly concentrated in Asia which accounts for yearly $75 \%$ of all affected cases. In India prevalence of low birth weight has been reported as $26 \%$ while the proportion of FGR has been found to be $54 \%$. 7,11

From a clinical point of view, the distinction between FGR versus SGA is mandatory because of the correlation with perinatal outcomes. It is reasonable to deliver electively FGR when lung maturation can be presumed (by 34 completed weeks) or earlier if signs of fetal deteriorations are observed. ${ }^{1,4,7,9}$ On the contrary, SGA fetuses are 
associated with virtually normal perinatal outcome and is generally considered that active management or elective delivery before full term offers no benefits. $1,2,4,7,9$

Globally, population control studies and case reports on FGR, its management and perinatal outcome have been extensively published in the western literature. In the Indian population, only few hospital-based population studies are reported on the management of FGR. Hence, this study will prove to be important in elucidating the effective management of FGR according to protocol in this part of the world. ${ }^{11,13}$

This study strives to illuminate the perinatal outcomes of stage-based management of FGR and determine the association between maternal sociodemographic profile and medical conditions with FGR in a tertiary hospital in Kerala, India.

\section{METHODS}

A descriptive study was conducted at the department of obstetrics and gynecology, govt. T. D. medical college, Alappuzha, between January 2018-2019. 320 antenatal women whose estimated fetal weight was $<10^{\text {th }}$ centile was selected. Convenience sampling procedure was adopted for selection of patients. They were enrolled into the study according to the inclusion criteria. They were educated regarding all aspects of the study and an informed written consent was taken. Individual pro forma was filled which included baseline variables such as age, parity, socioeconomic status, present and past obstetric history, maternal weight gain, coexistent maternal medical condition, previous USG and Doppler findings etc. They were categorized according to the Barcelona classification. Periodic follow up with serial USG and Doppler was done and managed as per the stage of FGR. Labour was induced according to the cervix favorability using Bishop's score. Perinatal outcomes in terms of birth weight/ mode of termination of pregnancy/ period of gestation/consideration of prenatal steroids coverage/Apgar score/ICU admissions and neonatal complications like low birth weight, hyaline membrane disease, intraventricular hemorrhage, bronchopulmonary dysplasia, pulmonary hemorrhage, hypothermia, hypoglycemia, hyperbilirubinemia, neonatal death if any were recorded and compiled. Both the baby and mother were followed up until discharge from hospital.

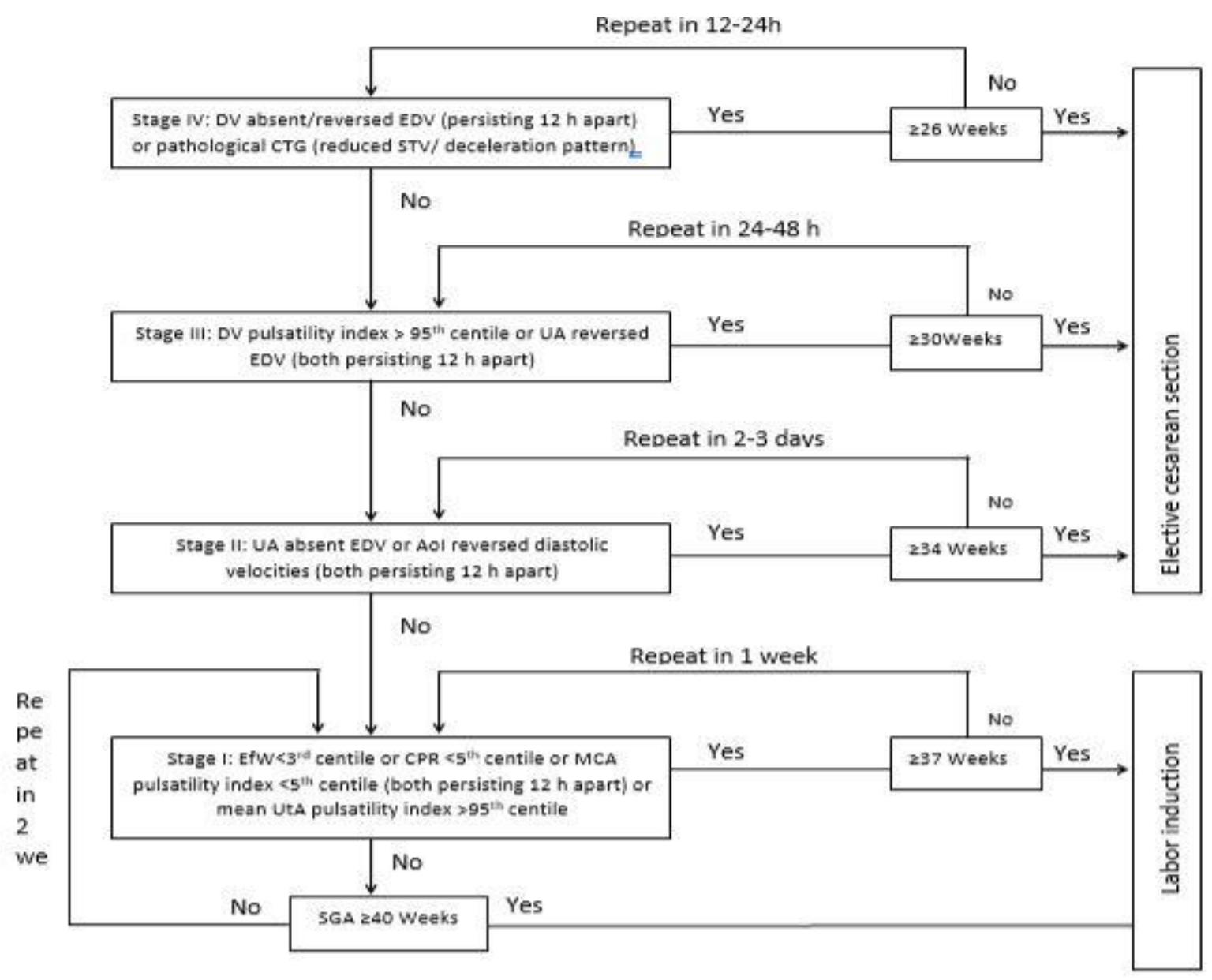

Figure 1: Stage-based classification and management of FGR., ${ }^{1,4,7}$ 
The data obtained were compiled and the same analyzed with SPSS software 16 using percentages and proportions. Frequencies were generated, variables compared and test of significance carried out using chi square. A $p<0.05$ was considered statistically significant.

Permission to conduct the research study was obtained from institutional research committee and institutional ethics committee of govt. T. D. medical college hospital, Alappuzha, Kerala, India.
The most commonly involved age group was 20-24 years, and the least involved $>35$ years. $33 \%$ were underweight accounting for the major cause of non-pathological forms of FGR-SGA. Over-weight population, some of them being overtly diabetic, can develop FGR due to placental insufficiency. One fourth of them had inadequate weight gain during pregnancy, which was statistically significant. $42 \%$ had short inter pregnancy interval-less than 2 years, predisposing to FGR due to inadequate replenishment of maternal energy sources.

\section{RESULTS}

Table 1: Association of selected variables based on stage.

\begin{tabular}{|c|c|c|c|c|c|c|c|}
\hline Variables & & $\begin{array}{l}\text { Small for } \\
\text { gestational age }\end{array}$ & $\begin{array}{l}\text { Stage } 1 \\
\text { FGR }\end{array}$ & Stage 2 & $\begin{array}{l}\text { Stage } 3 \\
\text { and } 4\end{array}$ & $\chi^{2}$ & $\mathbf{P}$ \\
\hline \multirow{5}{*}{ Age (Years) } & $<20$ & $13(31)$ & $26(61.9)$ & $3(7.1)$ & $0(0)$ & \multirow{5}{*}{16.47} & \multirow{5}{*}{0.171} \\
\hline & $20-24$ & $46(46.9)$ & $46(46.9)$ & $5(5.1)$ & $1(1)$ & & \\
\hline & $25-29$ & $40(44.4)$ & $42(46.7)$ & $5(5.6)$ & $3(3.3)$ & & \\
\hline & $30-34$ & $34(49.3)$ & $26(37.7)$ & $3(4.3)$ & $6(8.7)$ & & \\
\hline & $>35$ & $7(30.4)$ & $13(56.5)$ & $2(8.7)$ & $1(4.3)$ & & \\
\hline \multirow{4}{*}{ BMI $\left(\mathrm{kg} / \mathrm{m}^{2}\right)$} & Under weight & $53(49.5)$ & $47(43.9)$ & $6(5.6)$ & $1(0.9)$ & \multirow{4}{*}{13.76} & \multirow{4}{*}{0.131} \\
\hline & Normal & $45(42.5)$ & $54(50.9)$ & $5(4.7)$ & $2(1.9)$ & & \\
\hline & Over weight & $36(42.4)$ & $37(43.5)$ & $5(5.9)$ & $7(8.2)$ & & \\
\hline & Obese & $6(25)$ & $15(62.5)$ & $2(8.3)$ & $1(4.2)$ & & \\
\hline \multirow{2}{*}{$\begin{array}{l}\text { Weight gain } \\
\text { in pregnancy } \\
(\mathrm{kg})\end{array}$} & $<9$ & $25(31.3)$ & $41(51.3)$ & $7(8.8)$ & $7(8.8)$ & \multirow[b]{2}{*}{14.74} & \multirow[b]{2}{*}{0.002} \\
\hline & $>9$ & $115(47.5)$ & $112(46.3)$ & $11(4.5)$ & $4(1.7)$ & & \\
\hline \multirow{2}{*}{$\begin{array}{l}\text { IP interval } \\
\text { (Years) }\end{array}$} & $<2$ & $36(37.5)$ & $50(52.1)$ & $6(6.3)$ & $4(4.2)$ & \multirow{2}{*}{3.16} & \multirow{2}{*}{0.367} \\
\hline & $>2$ & $35(50)$ & $30(42.9)$ & $2(2.9)$ & $3(4.3)$ & & \\
\hline
\end{tabular}

Table 2: Association of perinatal outcome based on stage of FGR.

\begin{tabular}{|c|c|c|c|c|c|c|c|}
\hline \multicolumn{2}{|c|}{ Perinatal outcome } & $\begin{array}{l}\text { Small for } \\
\text { gestational age }\end{array}$ & $\begin{array}{l}\text { Stage } 1 \\
\text { FGR }\end{array}$ & Stage 2 & $\begin{array}{l}\text { Stage } 3 \\
\text { and } 4\end{array}$ & $\chi^{2}$ & $\mathbf{P}$ \\
\hline \multirow{4}{*}{$\begin{array}{l}\text { Gestational } \\
\text { age at which } \\
\text { pregnancy } \\
\text { terminated } \\
\text { (Weeks) }\end{array}$} & $>37$ & $137(53.3)$ & $119(46.3)$ & $1(0.4)$ & $0(0)$ & \multirow{4}{*}{236.4} & \multirow{4}{*}{$<0.01$} \\
\hline & $34-37$ & $3(7.5)$ & $27(67.5)$ & $10(25)$ & $0(0)$ & & \\
\hline & $28-32$ & $0(0)$ & $6(27.3)$ & $7(31.8)$ & $9(40.9)$ & & \\
\hline & $<28$ & $0(0)$ & $1(33.3)$ & $0(0)$ & $2(66.7)$ & & \\
\hline \multirow{5}{*}{$\begin{array}{l}\text { Birth weight } \\
\text { (kg) }\end{array}$} & 1 & $0(0)$ & $2(33.3)$ & $0(0)$ & $4(66.7)$ & \multirow{5}{*}{232.3} & \multirow{5}{*}{$<0.01$} \\
\hline & $1-1.5$ & $1(4.5)$ & $4(18.2)$ & $11(50)$ & $6(27.3)$ & & \\
\hline & $1.5-2$ & $4(13.8)$ & $21(72.4)$ & $3(10.3)$ & $1(3.4)$ & & \\
\hline & $2-2.5$ & $55(48.2)$ & $55(48.2)$ & $4(3.5)$ & $0(0)$ & & \\
\hline & $>2.5$ & $80(53)$ & $71(47)$ & $0(0)$ & $0(0)$ & & \\
\hline \multirow{10}{*}{$\begin{array}{l}\text { Neonatal } \\
\text { complication }\end{array}$} & Nil significant & $79(55.6)$ & $63(44.4)$ & $0(0)$ & $0(0)$ & 32.04 & $<0.01$ \\
\hline & Low birth weight & $51(33.1)$ & $76(49.4)$ & $17(11)$ & $10(6.5)$ & 31.36 & $<0.01$ \\
\hline & ARDS & $14(20)$ & 34 (48.6) & $12(17.1)$ & $10(14.3)$ & 63.65 & $<0.01$ \\
\hline & $\begin{array}{l}\text { Intraventricular } \\
\text { hemorrhage }\end{array}$ & $1(20)$ & $1(20)$ & $3(60)$ & $0(0)$ & 28.52 & $<0.01$ \\
\hline & $\begin{array}{l}\text { Necrotizing } \\
\text { enterocolitis }\end{array}$ & $0(0)$ & $5(38.5)$ & $4(30.8)$ & $4(30.8)$ & 51.16 & $<0.01$ \\
\hline & Sepsis & $2(6.5)$ & $14(45.2)$ & $8(25.8)$ & $7(22.6)$ & 72.81 & $<0.01$ \\
\hline & Hypothermia & $0(0)$ & $1(100)$ & $0(0)$ & $0(0)$ & 1.11 & 0.775 \\
\hline & Hypoglycemia & $2(28.6)$ & $4(57.1)$ & $0(0)$ & $1(14.3)$ & 3.38 & 0.337 \\
\hline & Hyperbilirubinemia & $1(9.1)$ & $8(72.7)$ & $1(9.1)$ & $1(9.1)$ & 5.94 & 0.114 \\
\hline & Anomaly & $0(0)$ & $0(0)$ & $3(100)$ & $0(0)$ & 51.1 & $<0.01$ \\
\hline
\end{tabular}


The most common medical co morbid illness was hypothyroidism-which in actual sense is associated with FGR, oligamnios, abortion, hypertensive disorders of pregnancy, GDM, PPH etc., followed by hypertension complicating pregnancy-4\%, diabetes complicating-4.3\%, auto immune and heart disease complicating pregnancy$3 \%$, infertility treated $-1.9 \%$. Among the obstetric co morbidities, gestational hypertension and preeclampsia spectrum accounts for the most common etiology for FGR, $5.3 \%$ and $5.6 \%$ respectively. In our study population, $6.2 \%$ were exposed to passive smoking. There was no exposure to alcohol or addictive drugs. $23.2 \%$ had history of first trimester abortion, 3\% had h/o GHTN. 3.7\% had h/o thyroid disease, GDM in $2.4 \%$.

$79.8 \%$ of the pregnancies were followed up and terminated $>37$ weeks of gestation, $12.4 \%$ between $34-37$ weeks, $7.7 \%$ before 32 weeks.

$46.9 \%$ of the birth weight was $>2.5 \mathrm{~kg}$, extremely low birth weight $-1.9 \%$, very low birth weight- $8.7 \%$, low birth weight $-53.7 \%$. Among the neonatal complications, low birth weight accounted for the maximum of $47.8 \%$, followed by ARDS-21.7\%, sepsis-9.6\%, necrotizing enterocolitis-4\%, neonatal death-1.56\%. 5.62\% of the pregnancies were terminated before 32 weeks, all of them belonging to stage 2, 3 and 4 of FGR. 7 cases of stage 2 FGR, 11 cases of stage 3 and 4 combined. Very low birth weight and extremely low birth weight were of stages 3 and 4 FGR. $13.4 \%$ of the population had h/o threatened abortion in first trimester. $10.3 \%$ had history of reduced fetal movements in third trimester. $10.3 \%$ had early onset FGR and the rest were late onset FGR. Among the maternal co morbid illness, hypertensive disorders of pregnancy had significant $\mathrm{p}<0.01$, which was also clinically significant.

\section{DISCUSSION}

Incidence of FGR was $15.23 \%$ in our study. According to national perinatal database, the incidence of FGR is said to be $9.65 \%$ among new born. According to Chandra and Mathews et al, incidence of FGR was $14.1 \%$. According to Shenoy et al, the incidence of FGR in south Kerala is $10.35 \%$. The increase in incidence in our study was probably due to the rural domicile and lower socioeconomic strata in the majority of the population. ${ }^{23-26}$

Small for gestational age or non-pathological forms of FGR accounted to $43 \%$, stage 1 FGR-47\%, stage 2 FGR$0.068 \%$, stage 3 FGR- $0.025 \%$, stage 4 FGR- $0.012 \%$.

\section{Maternal socio demographic factors}

Majority of the mothers were belonging to the age group between $20-24$ years followed by $25-29$ years. (30.4\% and $28 \%$ respectively), almost coming close to the study results of Shenoy et al, where 25-29 years was the frequently involved age group- $43.9 \% .^{26,28}$
Majority of the population were multigravida-55.7\% (178 cases), similar to the study of Shenoy et al and Satyavrathan et al. ${ }^{26,28} 47$ mothers had a history of previous abortion accounting for $14.6 \% .^{36}$

Among the multigravida, FGR recurred in $16.7 \%$, accounting to 53 cases. ${ }^{37} \mathrm{~A}$ total of 41 cases $(13 \%)$ were referred to our institution for expert management of FGR with or without maternal co morbidities. $44 \%$ of study population belonged to the class 4 and 5 of Kuppuswamy's modified socio-economic scale. $28.2 \%$ belonged to the class $3 .^{23}$

$33.2 \%$ of the mothers were underweight and $32.9 \%$ had BMI of normal range. $24.8 \%$ had inadequate weight gain during pregnancy $(<9 \mathrm{~kg}) .^{22}$

Among multigravida, 57.8\% (96 cases) had inadequate birth spacing of less than 2 years of inter pregnancy interval, which was statistically significant, most likely due to being less well educated, poor living conditions. ${ }^{11,12,14}$

\section{Medical, obstetric and personal history}

In our study population, $6.2 \%$ (20 cases) were exposed to passive smoking. ${ }^{12,15}$

Among maternal comorbid illnesses, hypertensive disorders in pregnancy accounted for the maximum-17.1\% (55 cases) including Gestational hypertension, preeclampsia, eclampsia, HELLP syndrome, chronic hypertension, diabetes complicating-10\%, thyroid disorders-7.3\%, anaemia complicating and heart disease complicating-3.2\%, infertility treated were $1.9 \% \quad(8$ cases). ${ }^{17,18,29,35-37}$

According to Shenoy et al, hypertensive disorders accounted for $24.3 \%$, thyroid disorders $-21.5 \%$, diabetes complicating-14.6\% were noted in FGR mothers. ${ }^{26}$ Similar results were obtained by Satyavrathan et al and Sharma et al and Motghare et al described gestational hypertension and anemia as maternal determinants of FGR. ${ }^{26,28,29,36}$

$25.6 \%$ ( 82 cases) had the associated oligamnios (AFI $<8$ $\mathrm{cm})^{30}$

In our study, 49 cases gave history of threatened abortion $(15.31 \%)$ and 33 cases perceived reduced fetal movements $(10.3 \%)$, none of which were statistically significant.

\section{FGR detection-follow up-delivery}

163 cases of FGR accounted for late onset FGR approximately $91.5 \% .27$ cases accounted for early onset FGR-8.4\%. Out of which, 6 cases were detected prior to 28 weeks of gestation. 
$79.8 \%$ of the pregnancies were followed up and terminated $>37$ weeks of gestation. $12.4 \%$ were terminated between 34-37 weeks. $7.7 \%$ of the population were terminated before 32 weeks. $0.9 \%$ ( 3 cases) were terminated before 28 weeks.

The mean diagnosis to delivery interval: $<48$ hours- $4.3 \%$ ( 14 cases), <1 week-13.4\% (43 cases), >1 week-82\% (260 cases)

Decision to deliver them was taken if 1) gestational age of 37 weeks, 2) absent end diastolic flow, reverse end diastolic flow, 3) worsening of maternal condition like HELLP, imminent eclampsia, uncontrolled hypertension 4) oligohydramnios $(\mathrm{AFI}<5)$. (criteria 2-4 irrespective of the gestational age)

As with Seal et al and Shenoy et al, majority (64\%) of FGR were diagnosed between $32-34$ weeks in this study. ${ }^{26,29}$ In Lekshmi et al, $60 \%$ of the FGR were born to mothers $<37$ weeks and $29 \%<32$ weeks. The mean GA at FGR diagnosis in normal Doppler group was 35.19 weeks and 34.29 weeks in abnormal Doppler $(p=0.03)$ and was statistically significant. The mean GA at delivery in Doppler abnormal group was 32.2 weeks and 37 weeks in Doppler normal group, while this variable was not statistically significant. In Malhotra et al, GA at diagnosis was 26.4 weeks in abnormal Doppler group and 28 weeks in normal Doppler group $(\mathrm{p}=0.001){ }^{26}$

According to Shenoy et al study, $33 \%$ delivered in 48 hours, $39 \%$ in a week, and $28 \%$ waited $>14$ days. $^{26}$

\section{Mode of delivery}

Among Small for gestational age group, 62.4\% delivered vaginally and $33.6 \%$ underwent cesarean delivery. The common indications for cesarean delivery were fetal distress, meconium-stained amniotic fluid, previous cesarean delivery, failed induction.

Among the pathological forms of FGR, all cases of stages 2,3 and 4 were delivered by cesarean section. Only those belonging to stage 1 FGR were kept for vaginal delivery. Among them, 53.5\% underwent emergency cesarean section. The most common indications were non reassuring fetal heart rate, fetal distress, previous CS, meconium-stained amniotic fluid, failed induction, 2 cases of malpresentation, 2 cases of grade 1 abruptio placenta. ${ }^{26,29}$

\section{Birth weight}

$46.9 \%$ of the birth weight was $>2.5 \mathrm{~kg}$ extremely low birth weight-1.9\%, very low birth weight- $8.7 \%$, low birth weight-53.7\%. Mean birth weight among Doppler normal group was $2.41 \mathrm{~kg}$ and Doppler abnormal group was 2.03 $\mathrm{kg}$, a significant difference of approximately 290 gm. ${ }^{26-28}$

\section{Sex predilection}

Female babies were more likely to be growth restricted $(52.3 \%)$ compared to male babies consistent with the study by Seal et al and Shenoy et al. However statistically insignificant. ${ }^{26,29}$

\section{Neonatal outcome}

In our study, total NICU admissions were 183, accounting for $57.18 \%$. Majority of them were those with abnormal Doppler. All cases of abnormal Doppler were admitted to the NICU in view of risks of necrotizing enterocolitis, babies were kept nil per oral in view of possibility of mesenteric ischemia. The management of babies with abnormal Doppler varies according to the institutions' guidelines and neonatal set up.

The incidence of LBW-47.8\%, ARDS-21\%, sepsis-9.6\%, necrotizing enterocolitis-4\%, hyperbilirubinemia- $4.9 \%$. The incidence of congenital anomaly detected postnatally$0.9 \%$, with a single case of trisomy 21 , a single case of stillbirth-0.3\%. ${ }^{19,20}$ The incidence of neonatal death that occurred before discharge- $1.56 \%$, accounting for 5 cases, 3 of the cases belonged to stage 4 FGR and 2 cases of stage 3 FGR.

APGAR score less than or equal to 7 was seen in $8.7 \%$, accounting for 27 babies, all of them needed resuscitation in the form of mechanical ventilation or CPAP, carried an overall poor prognosis.

Gestational age at delivery and NICU stay were compared and it was found that neonates born at term had shorter NICU stays than very preterm neonates. Also, FGR with normal Doppler had shorter NICU stays than FGR neonates with Doppler abnormalities $(\mathrm{p}=0.003)$ similar to Rekha et al ( $\mathrm{p}=0.003)$. Bassetty et al found that gestational age at delivery was a strong determinant of neonatal outcome using obstetric Doppler. ${ }^{26,28,37}$

Neonatal hyperbilirubinemia $32.39 \%$, respiratory distress $21.12 \%$, sepsis $19.71 \%$ were seen in Baschat et al. According to Pallotto et al, perinatal asphyxia, hypothermia, hypoglycemia and hypocalcemia were common in IUGR babies. ${ }^{37}$

Abnormal BPP fetuses had significant Doppler pattern abnormalities. The inverse relationship between abnormal BPP scores and presence of fetal distress, FGR, NICU admissions were described by Manning et al. ${ }^{31}$ Cosmi et al showed that, in IUGR fetuses delivered at 32 weeks, the integration of Doppler and neonatal outcome was statistically correlated with BPP. Perinatal morbidity worsened with lower birthweights, Doppler abnormality, remote from term and hypertension. ${ }^{31,32}$

\section{Limitations}

The sample size was small. Many clinically significant parameters were not statistically significant. The period of study was short. The long-term sequelae of FGR like 
neurodevelopmental abnormalities, incidence of cerebral palsy, impaired cognitive function as a result of intrauterine hypoxia and acidosis, perinatal asphyxia which adversely affects the quality of life of the growing child could not be deduced from this study. Longer follow up of the mother and the child would be more informative.

\section{CONCLUSION}

The proportion of FGR among antenatal women in this study is $15.23 \%$, among which small for gestational age takes a lion's share. FGR is one of the most challenging issues in obstetrics with an evolving and continuous search for improvement in terms of definition, diagnosis and management. It is more prevalent in Asia and the developing parts of the world including India, accounting for approximately $75 \%$ of all affected infants. Hence, vigilant antepartum surveillance with Doppler ultrasound and evaluation of maternal risk factors is mandatory. Prompt intervention in the event of worsening fetal or maternal condition is imperative. This study sheds light on the sociodemographic profile of obstetric population in rural Kerala. It also elucidates risk factors which can be used for screening of patients during the antenatal period to identify high risk women and subjecting them to vigilant monitoring thereby preventing unwarranted preterm termination of pregnancy and improving the overall perinatal outcome.

\section{ACKNOWLEDGMENTS}

Authors would like to thank department of obstetrics and gynecology and department of pediatrics, govt. T. D. medical college, Alappuzha for their help and guidance during the study.

Funding: No funding sources Conflict of interest: None declared

Ethical approval: The study was approved by the Institutional Ethics Committee

\section{REFERENCES}

1. Mureșan D, Rotar IC, Stamatian F. The usefulness of fetal Doppler evaluation in early versus late onset intrauterine growth restriction. Review of the literature. Med Ultrason. 2016;18(1):103-9.

2. Cohen E, Baerts W, van Bel F. Brain-Sparing in Intrauterine Growth Restriction: Considerations for the Neonatologist. Neonatology. 2015;108(4):269-76.

3. Meher S, Hernandez-Andrade E, Basheer SN, Lees C. Impact of cerebral redistribution on neurodevelopmental outcome in small-forgestational-age or growth-restricted babies: a systematic review. Ultrasound Obstet Gynecol. 2015;46(4):398-404.

4. Figueras F, Gratacós E. Update on the diagnosis and classification of fetal growth restriction and proposal of a stage-based management protocol. Fetal Diagn Ther. 2014;36(2):86-98.
5. Senat MV, Tsatsaris V. Prenatal management of isolated IUGR. J Gynecol Obstet Biol Reprod (Paris). 2013;42(8):941-65.

6. Perrotin F, Simon EG, Potin J, Laffon M. Delivery of the IUGR fetus. J Gynecol Obstet Biol Reprod (Paris). 2013;42(8):975-84.

7. Salomon LJ, Malan V. Managing and identifying the causes of IUGR. J Gynecol Obstet Biol Reprod (Paris). 2013;42(8):929-40.

8. Flamant C, Gascoin G. Short-term outcome and small for gestational age newborn management. J Gynecol Obstet Biol Reprod (Paris). 2013;42(8):985-95.

9. Unterscheider J, O’Donoghue K, Daly S, Geary MP, Kennelly MM, McAuliffe FM et al. Fetal growth restriction and the risk of perinatal mortality-case studies from the multi-centre PORTO study. BMC Preg Childbirth. 2014;14(1):63.

10. Fetal growth disorders. In Williams Obstetrics. $25^{\text {th }}$ ed. New York, NY: Mc Graw Hill. 2013:872-90.

11. Buck Louis GM, Grewal J, Albert PS. Racial/ethnic standards for fetal growth: the NICHD Fetal Growth Studies. Am J Obstet Gynecol. 2015;213:449.e1.

12. Unterscheider J, Daly S, Geary MP. Optimizing the definition of intrauterine growth restriction: the multicenter prospective PORTO Study. Am J Obstet Gynecol. 2013;208:290.e1.

13. Mlynarczyk M, Chauhan SP, Baydoun HA. The clinical significance of an estimated fetal weight below the $10^{\text {th }}$ percentile: a comparison of outcomes of $<5^{\text {th }}$ vs $5^{\text {th }}-9^{\text {th }}$ percentile. Am J Obstet Gynecol. 2017;217:198.e1.

14. Barker ED, McAuliffe FM, Alderdice F. The role of growth trajectories in classifying fetal growth restriction. Obstet Gynecol. 2013;122:248.

15. Gardosi J. New definition of small for gestational age based on fetal growth potential. Horm Res. 2006;65(3):15.

16. Gardosi J, Figueras F, Clausson B, Francis A. The customised growth potential: an international research tool to study the epidemiology of fetal growth. Paediatr Perinat Epidemiol. 2011;25:2.

17. Figueras F, Gardosi J. Intrauterine growth restriction: new concepts in antenatal surveillance, diagnosis, and management. Am J Obstet Gynecol. 2011;204:288.

18. Mateus J, Newman RB, Zhang C. Fetal Growth Patterns in Pregnancy-Associated Hypertensive Disorders: NICHD Fetal Growth Studies. Am J Obstet Gynecol. 2019.

19. Mendez H. Introduction to the study of pre- and postnatal growth in humans: a review. Am J Med Genet. 1985;20:63

20. Khoury MJ, Erickson JD, Cordero JF, McCarthy BJ. Congenital malformations and intrauterine growth retardation: a population study. Pediatrics. 1988;82:83.

21. Gülmezoglu AM, Hofmeyr GJ. Betamimetics for suspected impaired fetal growth. Cochrane Database Syst Rev. 2001;CD000036.

22. Morris RK, Malin G, Robson SC. Fetal umbilical artery Doppler to predict compromise of 
fetal/neonatal wellbeing in a high-risk population: systematic review and bivariate meta-analysis. Ultrasound Obstet Gynecol. 2011;37:135.

23. Fanaroff AA, Hack M, Walsh MC. The NICHD neonatal research network: changes in practice and outcomes during the first 15 years. Semin Perinatol. 2003;27(4):281-7.

24. De Onis M, Blossner M, Villar J. Levels and patterns of intrauterine growth retardation in developing countries. Eur J Clin Nutr. 1998;52(1):S5-S15.

25. Chandra S, Mathews SC. Perinatal morbidity and mortality in low birthweight babies. J Obstet Gynaecol India. 2003:53(3):237.

26. Shenoy HT, James SX, Shenoy ST. Maternal risk factors and perinatal outcomes in fetal growth restriction using obstetric Doppler in South Kerala, India. Int J Reprod Contracept Obstet Gynecol. 2019;8(1):6-13.

27. Singh A, Ambujam K. Maternal socio-demographic determinants and fetal outcome of intrauterine growth restriction. Int J Reprod Contracept Obstet Gynecol. 2018;7:3843-7.

28. Satyavrathan V, Ahmed N, Sundrappa S. Study of perinatal outcomes of pregnancies with intrauterine growth restriction in a tertiary care centre in North Kerala. J Evid Based Med Healthc. 2017;4(37):22038.

29. Seal A, Dasgupta A, Sengupta M, Dastider R, Sen S. Analysis of fetal growth restriction in pregnancy in subjects attending in an obstetric clinic of a tertiary care teaching hospital. Int J Reprod Contracept Obstet Gynecol. 2018;7:973-80.

30. Rutherford SE, Phelan JP, Smith CV, Jacobs N. The four-quadrant assessment of amniotic fluid volume: an adjunct to antepartum fetal heart rate testing. Obstet Gynecol. 1987;70(3 Pt 1):353-6.
31. Manning FA, Harman CR, Morrison I, Menticoglou SM, Lange IR, Johnson JM. Fetal assessment based on fetal biophysical profile scoring. IV. An analysis of perinatal morbidity and mortality. Am J Obstet Gynecol. 1990;162(3):703-9.

32. Cosmi E, Ambrosini G, D'antona D, Saccardi C, Mari G. Doppler, cardiotocography, and biophysical profile changes in growth restricted fetuses. Obstet Gynecol. 2005;106:1240-5.

33. Turan OM, Turan S, Gungor S, Berg C, Moyano D, Gembruch $U$ et al. Progression of Doppler abnormalities in intrauterine growth restriction. Ultrasound Obstet Gynecol. 2008;32:160-7.

34. Cochran WD, Lee KG. Assessment of the new-born. Manual of Neonatal Care. $5^{\text {th }}$ ed. Philadelphia: Lippincott Williams and Wilkins. 2004.

35. Ghazi HA, Spielberger C, Kallen B. Delivery outcome after infertility: a registry study. Fertil Steril. 1991;55:726-32.

36. Motghare DD, Frederick SV, Pawaskar M, Kulkarni. Maternal determinants of intrauterine growth restriction in Goa, India: a case-control study. Global J Medic Public Health. 2014;3(1):08.

37. Bassetty KC, Phukan P, Ahmed RD, Borah R. Outcomes of Neonates in Pregnancies with Intrauterine Growth Restriction in Developing Countries: A Cross-sectional Study Over a Period of 6 Months. Gynecol Obstet (Sunnyvale). 2017;7:434.

Cite this article as: Rangarajan B, Karunakaran L. Perinatal outcome of stage-based management of fetal growth restriction in a government tertiary care hospital in Kerala, India. Int J Reprod Contracept Obstet Gynecol 2021;10:2354-61. 